\title{
Using Immune Genetic Algorithm to Optimize BP Neural Network and Its Application
}

\author{
Peng-fei LIU ${ }^{1}$, Qun-tai SHEN ${ }^{1}$ and Jun ZHI ${ }^{2, *}$ \\ ${ }^{1}$ School of Information Science and Engineering, Central South University, China \\ ${ }^{2}$ Beijing institute of remote sensing information, China \\ ${ }^{*}$ Corresponding author
}

Keywords: Immune genetic algorithm, Neural network, Image recognition

\begin{abstract}
It proposes a target classification and recognition method based on immune genetic neural network algorithm. Immune genetic algorithm is adopted to optimize the initial weights and thresholds of BP (Back Propagation) network. Respectively use traditional BP neural network algorithm and immune genetic neural network algorithm to train network, until the convergence error precision of neural network reaches a pre-set requirement. Simulation results show that with the same hidden layer nodes and error precision request, it proposes immune genetic neural network algorithm for image recognition, its effect is better than that of traditional BP algorithm, improves the convergence speed of BP neural network, and reduces the training time. The recognition rate improves to some extent.
\end{abstract}

\section{Immune Genetic Algorithm}

Genetic algorithm is a stochastic search algorithm, groups search and information exchanges between individuals are achieved through crossover and mutation operators [1]. It ensures the entire population evolutionary direction under the "survival of the fittest" selection mechanism. But crossover and mutation operators are carrying out the random iterative search under certain probability conditions. In the evolution of each individual, but also it may produce degradation.

Immunization is a phenomenon of organisms autonomously against viruses to maintain their physical activity stable and equilibrium. When the immune system produces "initial immune response" with a specific antigen, part of the antibodies become memory cells, and remember the antigen [2]. When they meet again, it will produce "twice immune response" and clear the antigen quickly. Using the remembered antigen recognition function in the genetic algorithm, it can speed up the search.

Immune genetic algorithm combines immune algorithm with genetic algorithm, make the objective function of practical problem correspond to an antigen, problem solution correspond to the antibody; use antigen and antibody affinity to represent the approximation degree of feasible solution and optimal solution [3]. For a particular problem, the algorithm randomly generates a set of initial antibody, and then calculates the fitness of each antibody, achieve the environmental adaptation by promotion and restrain between antibodies.

\section{BP Neural Network}

BP neural network is also known as back propagation neural network. It is divided into the input layer, intermediate layer (hidden layer) and output layer [4]. There is no connection between each layer of neurons; the input layer and output layer are 
connected with the outside world. The BP neural network algorithm is a kind of supervised learning algorithm.

\section{Method of Immune Genetic Algorithm to Optimize BP Neural Network Weights}

Traditional BP network is sensitive to the initial weights and threshold [5]. There are defects of slow convergence speed and easy convergence to the local minimum. Immune genetic algorithm has stronger global searching ability. It can converge to the global optimal solution, and it is robust. A combination of both can well overcome network deficiencies and make BP network have a faster convergence and stronger learning ability. Therefore, immune genetic algorithm is adopted to optimize the initial weights and thresholds of BP network. The optimization process of immune genetic algorithm includes individual coding and decoding, fitness calculation, promotion and inhibition of antibody concentration, genetic update of antibody population. The concrete method is as follows.

\section{Antibody Gene Coding and Decoding}

Real coding has the advantage of high precision and large space search. Compared with binary coding, coded decimal conversion step is omitted in the real coding. Coding is simple and intuitive; search efficiency is improved. Therefore, weights and thresholds between the neurons of BP network are encoded with real number coding method. It directly takes weights and thresholds as the value of individual genes.

Assuming that neurons of input layer, hidden layer and output layer in the BP network are respectively $n, m, l$, weight between input layer and hidden layer is $w_{i j}$, weight between hidden layer and output layer is $w_{j k}$, threshold of hidden layer is $b_{j}$, threshold of output layer is $b_{k}$, where, $i, j, k$ respectively expresses the index number of input layer node, hidden layer node and output layer node. So the coding method for population individuals is ${ }_{\left(w_{11} \cdots w_{i j} \cdots w_{m n}, b_{1} \cdots b_{j} \cdots b_{m}, w_{11} \cdots w_{j k} \cdots w_{m l}, b_{1} \cdots b_{k} \cdots b_{l}\right)}$, each individual represents a kind of initial weights and thresholds scheme of BP network.

The individual is coded according to the sequence of weights and thresholds, so when decoding, we just need to read corresponding number of genes for population individuals. For example, $w_{i j}$ reads former $m \times(i-1)+j$ genetic data, $b_{j}$ reads $m \times n+j$ genetic data.

\section{Design of Antibody Fitness Function}

Fitness function of immune genetic algorithm is used to determine the adaption of population individuals during the optimization process. Fitness function directly determines the optimal solution. Using immune genetic algorithm to optimize BP network, the final results should be combined with the output of BP network. So firstly we consider using error performance function of BP network as the fitness function of evolutionary individuals.

Set that the number of antibody population is $n$. Each antibody corresponds to a kind of network, as a combined network structure. Use error performance function to calculate the corresponding error of each antibody.

Fitness function $F\left(x_{i}\right)$ is defined as the error performance function of neural network, that is $E\left(x_{i}\right)$. Namely: 


$$
F\left(x_{i}\right)=\frac{1}{E\left(x_{i}\right)+\xi} \text {. }
$$

Where

$$
E\left(x_{i}\right)=\frac{1}{2 P} \sum_{n=1}^{P} \sum_{j=1}^{S}\left(T_{j}^{n}-Y_{j}^{n}\right)^{2} .
$$

$P$ is the total number of training samples; $S$ is the output layer nodes of BP neural network; $Y_{j}^{n}$ and $T_{j}^{n}$ are respectively the actual output and expected output of No.n training sample on the No.j output node. $\xi$ is a constant greater than 0 .

\section{Concentration of the Antibody}

Make the parameter to be designed mapped for the allele $j$ in antibody $i$, it is expressed as $G^{j}(i) . n$ antibodies constitute a non-empty immune system set $X$. Set $d_{i}$ as the distance measurement between antibody $i$ and other antibodies in the collection $X$.

$$
d_{i}=\sum_{k} \sqrt{\sum_{j}\left(G^{k}(i)-G^{k}(j)\right)^{2}} .
$$

Concentration of antibody $i$ is inversely proportional to $d_{i}$, set antibody concentration $A_{i}$ as follows.

$$
A_{i}=\frac{1}{1+d_{i}}
$$

The larger $A_{i}$ is, the more similar antibodies there are in the collection $X$, otherwise $A_{i}$ is smaller.

\section{Promotion and Inhibition of the Antibody}

Introduce the concentration factor to adjust the probability of antibody concentration. Suppress the antibodies with high concentration and low affinity; promote the antibodies with low concentration and high affinity. Probability selection formula based on the antibody concentration is as follows.

$$
P(i)=\alpha\left(1-A_{i}\right) F(i) \text {. }
$$

Where, $\alpha$ is an adjustable parameter between 0 and 1 . In the collection $X$, the larger the antibody concentration is, the lower the affinity is, and the smaller the selection probability is. Otherwise the larger the selection probability is.

\section{Genetic Update of Antibody Population}

(1) Crossover. Including cross way and cross probability. In each generation of the population, the higher the crossover probability is, the faster the new structure introduces. Carry on the crossover to the individuals $G_{i}$ and $G_{i+1}$ with probability $P_{c}$, we can get new individuals $G_{i}^{\prime}$ and $G^{\prime}{ }_{i+1}$. Directly copy the individuals of no crossover operation.

(2) Mutation operation. When the fitness change is too small, it needs to increase the mutation probability, and increase the diversity of samples, so that the population can 
jump out of local minimum. When evolution meets the requirements, remain the mutation probability. Mutation probability adaptively changes according to the fitness variation and evolution generations. The selection of mutation point is determined by population diversity measure. Mutate the individual $G_{j}$ and generate $G^{\prime}{ }_{j}$ with probability $P_{m}$. Diversity measures of population and mutation probability are defined as follows.

$$
\begin{aligned}
& m(i)=1-\frac{1}{n}\left(\max \left\{\sum_{j=1}^{n} a_{l j}, \sum_{j=1}^{n}\left(1-a_{l j}\right)\right\}-\min \left\{\sum_{j=1}^{n} a_{l j}, \sum_{j=1}^{n}\left(1-a_{l j}\right)\right\}\right) . \\
& p(i)=\frac{m(i)}{\sum_{i=1}^{l} m(i)} .
\end{aligned}
$$

Where, $m(i)$ is the diversity measure of No.i. $l$ is the total number of genes.

\section{Termination Condition Judgment}

Output the optimization results. Here the termination condition is that the error between the algorithm calculation result and the actual result is less than a certain fixed value.

\section{Simulation Analysis}

Coil-20 (Columbia object image library) image database contains 20 objects. Firstly fix the camera, and then put the object on a rotating platform. Take the pictures at intervals of $5^{\circ}$, and get object images of 72 different perspectives. Each object only lists three images of different view angle after translation, rotation and scaling. Each object represents a class, the sample is divided into 3 classes, and each class has 72 images. Use 36 images of them to train the neural network, the rest 36 images test the trained neural network.

Separately use 36 sample images of each type to test the immune genetic neural network algorithm and the traditional BP neural network algorithm in order to identify the recognition effect of the network as shown in Table1.

Table 1: Recognition rate comparison of immune genetic neural network algorithm and the traditional

\begin{tabular}{|c|c|c|c|c|c|}
\hline \multirow[b]{2}{*}{ sample } & \multirow{2}{*}{$\begin{array}{l}\text { training sample } \\
\text { / total samples }\end{array}$} & \multicolumn{2}{|c|}{ correct identification / total recognition } & \multicolumn{2}{|c|}{ Recognition rate $(\%)$} \\
\hline & & $\begin{array}{l}\text { traditional BP neural } \\
\text { network algorithm }\end{array}$ & $\begin{array}{c}\text { immune genetic neural } \\
\text { network algorithm }\end{array}$ & $\begin{array}{l}\text { traditional BP neural } \\
\text { network algorithm }\end{array}$ & $\begin{array}{l}\text { immune genetic neural } \\
\text { network algorithm }\end{array}$ \\
\hline Obj1 & $36 / 72$ & $32 / 36$ & $34 / 36$ & 88.9 & 94.4 \\
\hline Obj2 & $36 / 72$ & $31 / 36$ & $33 / 36$ & 86.1 & 91.7 \\
\hline Obj3 & $36 / 72$ & $30 / 36$ & $32 / 36$ & 83.3 & 88.9 \\
\hline
\end{tabular}
BP neural network algorithm

It can be seen: it proposes immune genetic neural network algorithm for image recognition, its effect is better than that of traditional BP algorithm, improves the convergence speed of BP neural network, and reduces the training time. On the recognition rate, compared with the traditional $\mathrm{BP}$ algorithm, immune genetic neural network algorithm improves to some extent. But when the object scales down to $1 / 4$ 
or more, the recognition rate decreases, the main reason is that the target in the rotation, scaling distortion.

\section{Reference:}

[1] W. Huang and L. Jiao. Artificial Immune Kernel Clustering Network for Unsupervised Image Segmentation. Progress in Natural Science, 2008.

[2] A.J. Graaff and A. P. Engelbrecht. Using Sequential Deviation to Dynamically Determine the Number of Clusters Found by a Local Network Neighbourhood Artificial Immune System. Applied Soft Computing Journal, 2011.

[3] M. Bereta. Immune K-means and Negative Selection Algorithms for Data Analysis. Information Sciences, 2009.

[4] S. Gou. Multi-elitist Immune Clonal Quantum Clustering Algorithm. Neurocomputing, 2013.

[5] R. Liu. Immunodomaince Based Clonal Selection Clustering Algorithm. Applied Soft Computing, 2012.

[6] J. Wang. Study of Optimizing Method for Algorithm of Minimum Convex Closure Building for 2D Spatial Data. Acta Geodaetica et Cartographica Sinica, 2002. 